\begin{tabular}{|l|l|l|}
\hline \multicolumn{2}{|c|}{ PublisherInfo } \\
\hline \hline PublisherName & $:$ & BioMed Central \\
\hline \hline PublisherLocation & $:$ & London \\
\hline \hline PublisherImprintName & $:$ & BioMed Central \\
\hline \hline
\end{tabular}

\title{
Tamoxifen treatment for DCIS - NSABP B-24 trial
}

\begin{tabular}{|l|l|l||}
\hline \multicolumn{2}{|c|}{ ArticleInfo } \\
\hline \hline ArticleID & $:$ & 3606 \\
\hline \hline ArticleDOI & $:$ & $10.1186 /$ bcr-1999-66585 \\
\hline \hline ArticleCitationID & $:$ & 66585 \\
\hline \hline ArticleSequenceNumber & $:$ & 26 \\
\hline \hline ArticleCategory & $:$ & Paper Report \\
\hline ArticleFirstPage & $:$ & 1 \\
\hline \hline ArticleLastPage & $:$ & 4 \\
\hline \hline & & RegistrationDate : 1999-6-15 \\
\hline ArticleHistory & $:$ & OnlineDate \\
\hline \hline ArticleCopyright & $:$ & Current Science Ltd1999-6-15 \\
\hline \hline ArticleGrants & $:$ & \\
\hline \hline ArticleContext & $:$ & 1305811 \\
\hline \hline
\end{tabular}




\section{Keywords}

breast, intraductal carcinoma, lumpectomy, radiotherapy, tamoxifen

\section{Introduction}

The management of ductal carcinoma in situ (DCIS) has changed markedly since the mid-1980s with many cases now being treated by breast conserving treatment. The National Surgical Adjuvant Breast and Bowel Project (NSABP) protocol B-17 clearly showed that lumpectomy followed by radiation therapy was more effective than lumpectomy alone in prevention of an invasive tumor in the ipsilateral breast. Tamoxifen has also been reported to prevent tumour recurrences in the ipsilateral breast and second primary tumors in the contralateral breast of women who have undergone lumpectomy and radiation therapy for primary invasive breast cancer.

\section{Aims}

To test the hypothesis that in patients with non-invasive DCIS, treatment with lumpectomy, postoperative radiation therapy, and tamoxifen would be more effective than lumpectomy and radiation therapy alone in the prevention of invasive and non-invasive cancers in the ipsilateral and contralateral breast.

\section{Comments}

This well designed and conducted study clearly shows that tamoxifen lowers the risk of breast cancerrelated events in this group of women. However, it would be inadvisable for most women with DCIS to receive adjuvant tamoxifen as DCIS is a heterogeneous disorder for which treatment should be tailored for the individual patient. Adjuvant tamoxifen probably represents overtreatment for most patients with DCIS, as most cases will not recur if treated with adequate local excision alone. The next step is to identify high risk patients that will benefit most from tamoxifen. This study already demonstrates that patients who have tumor positive resection margins or had DCIS associated with comedonecrosis have lower recurrence rates with the addition of tamoxifen. Further analysis of the pathological data may help identify other high-risk women. 


\section{Methods}

A total of 1804 women with DCIS, including those whose resected sample margins were involved with tumor, were randomly assigned lumpectomy, radiation therapy (50 Gy), and placebo $(n=902)$, or lumpectomy, radiation therapy, and tamoxifen ( $20 \mathrm{mg}$ daily for 5 years, $n=902)$. The women were also stratified by age, tumor type and method of detection of the tumour. The annual event rates and cumulative probability of invasive or non-invasive ipsilateral and contralateral tumours over 5 years were then compared.

\section{Results}

Of the 1804 women, 29 became ineligible after randomisation and 14 did not begin the assigned therapy. A further 269 in the placebo group and 295 in the tamoxifen group were not fully compliant. The patient characteristics were similar in the two groups, $65 \%$ were postmenopausal, $16 \%$ had positive resected sample margins after definitive surgery and more than $80 \%$ of the tumours had maximum dimensions of $1 \mathrm{~cm}$ or less.

At 5 years of follow-up, $83 \%$ of patients who received placebo were event?free compared with $87 \%$ of tamoxifen-treated patients. The placebo group had 130 invasive and non-invasive breast-cancer events in the ipsilateral breast, contralateral breast, or presented as metastases at regional or distant sites compared with 84 in the tamoxifen group. Women in the tamoxifen group had fewer breast-cancer events at 5 years than did those on placebo $(8.2$ versus $13.4 \%, \mathrm{P}=0.0009)$. The cumulative incidence of all invasive breast-cancer events in the tamoxifen group was $4.1 \%$ at 5 years: $2.1 \%$ in the ipsilateral breast, $1.8 \%$ in the contralateral breast, and $0.2 \%$ at regional or distant sites. In the tamoxifen group the estimated rate ratio represented $37 \%$ fewer breast-cancer events. The risk of ipsilateral-breast cancer was lower in the tamoxifen group even when sample margins contained tumor and when DCIS was associated with comedonecrosis. The 5 year survival was $97 \%$ for the two groups.

\section{Discussion}

Women with DCIS treated by lumpectomy and radiation therapy showed additional benefit from tamoxifen. The advantage was due mainly to a reduction in the rate of invasive cancer, especially in the ipsilateral breast. If the findings of the NSABP B-17 trial are also considered then the cumulative incidence of all breast-cancer-related events in women with DCIS treated by lumpectomy alone was about $25 \%$ at 5 years, $13 \%$ after the addition of radiation therapy and $8 \%$ when tamoxifen was also given. Thus tamoxifen and radiation therapy led to a $68 \%$ lower cumulative incidence of all breastcancer events at 5 years of follow-up than in women treated with lumpectomy alone in B-17. These findings contribute to the decision-making process about treatment of patients with mammographically 
detected DCIS when radiological or pathological evidence suggests that all of the cancer was not removed after lumpectomy. Moreover, the value of tamoxifen used in combination with radiation therapy to lower the occurrence of invasive cancer justifies the suggestion that combined therapy replaces mastectomy for the treatment of DCIS patients in whom radiological findings are unlikely to be related to an invasive tumour.

\section{References}

1. Fisher B, Dignam J, Wolmark N, Wickerham DL, Fisher ER, Mamounas E, Smith R, Begovic M, Dimitrov NV, Margolese RG, Kardinal CG, Kavanah MT, Fehrenbacher L, Oishi RH: Tamoxifen in treatment of intraductal breast cancer: National Surgical Adjuvant Breast and Bowel Project B-24 randomised controlled trial. Lancet. 1999, 353: 1993-2000. 